Bull. Astr. Soc. India (0000) 00, 000-000

\title{
Chandrasekhar and Modern Stellar Dynamics
}

\author{
N.W. Evans* \\ Institute of Astronomy, Madingley Rd, Cambridge, CBз OHA, UK
}

2018 May 21

\begin{abstract}
Stellar dynamics occupied Chandrasekhar's interest for a brief interlude between his more prolonged studies of stellar structure and radiative transfer. This paper traces the history of one of his ideas - namely, that the shape of the galactic potential controls the orientation of the stellar velocity dispersion tensor. It has its roots in papers by Eddington (1915) and Chandrasekhar (1939), and provoked a fascinating dispute between these two great scientists - less well-known than their famous controversy over the white dwarf stars. In modern language, Eddington claimed that the integral curves of the eigenvectors of the velocity dispersion tensor provide a one-dimensional foliation into mutually orthogonal surfaces. Chandrasekhar challenged this, and explicitly constructed a counter-example. In fact, the work of neither of these great scientists was without flaws, though further developments in stellar dynamics were to ultimately draw more on Eddington's insight than Chandrasekhar's. We conclude with a description of modern attempts to measure the orientation of the velocity dispersion tensor for populations in the Milky Way Galaxy, a subject that is coming into its own with the dawning of the Age of Precision Astrometry.
\end{abstract}

Keywords : celestial mechanics, stellar dynamics - galaxies: kinematics and dynamics - galaxies: general - Galaxy: stellar populations

\section{Introduction}

Chandrasekhar was perhaps the most influential theoretical astrophysicist of his time. This influence was particularly felt through an outstanding series of research monographs

*e-mail:nwe@ast.cam.ac.uk 
that continue to be read today. In fact, most astronomers first encounter Chandrasekhar through the cheap Dover reprints of books like Stellar Structure, Radiative Transfer, Hydrodynamic and Hydromagnetic Stability and Ellipsoidal Figures of Equilibrium. These books bristle with formulae, equations, numerical tables, graphs and historical notes, leavened with an immaculate prose style. They make exciting reading still today because they contain so much classic astrophysics so lucidly explained.

In his Nobel lecture, Chandraskhar (1984) has written " There have been seven periods in my life. They are briefly: 1) stellar structure, including the theory of white dwarfs (1929-1939); 2) stellar dynamics, including the theory of Brownian motion (1938-1943); 3) the theory of radiative transfer, the theory of the illumination and the polarization of sunlit sky (1943-1950); 4) hydrodynamic and hydromagnetic stability (1952-1961); 5) the equilibrium and stability of ellipsoidal figures of equilibrium (1961-1968); 6) the general theory of relativity and relativistic astrophysics (1962-71) and 7) the mathematical theory of black holes (1974-1983)."

So, Chandrasekhar's work on stellar dynamics occupied a brief interlude of time. It began in 1938 as an natural progression of his interests in the structure and evolution of stars. This was at the height of his famous controversy with Eddington over the fate of the white dwarf stars. It was over by 1943 , when Chandrasekhar was commuting between the Yerkes Observatory in Chicago and the Aberdeen Proving Ground in Maryland, working on ballistics as part of the war effort. His research interests had moved towards radiative transfer - the subject which Chandrasekhar himself has described as the one giving him most satisfaction (Wali 1991).

Chandrasekhar's (1943) book Principles of Stellar Dynamics is not as well-known or as magisterial as some of his others. The work on dynamical friction and dynamics of star clusters has proved to be of long-lasting value (see e.g., Heggie's article in this issue). However, much of the book reads oddly today. There are two long and, to modern eyes, puzzling chapters devoted to problems in collisionless stellar dynamics, in particular, galaxy models consistent with the ellipsoidal hypothesis. This term is not much used nowadays, but was introduced by Eddington (1915) as a generalisation of the triaxial Gaussian distribution of velocities used by Schwarzschild (1908) to describe the velocities of stars in the solar neighbourhood. This is the work we shall examine here, and it is fair to say that this is not Chandrasekhar at his most memorable. But, its connection with the earlier work of Eddington is fascinating, especially considering the personal relations between these two great scientists. And even when Chandrasekhar was not at his brilliant best, he could still find much of interest that others had overlooked.

So, we shall trace out the twists and turns that take us from the founding of stellar dynamics by Jeans and Eddington at the beginning of the twentieth century to modern times. Chandrasekhar himself contributed both fresh footpaths and blind alleys to this mazy route. 


\section{Eddington and the Ellipsoidal Hypothesis}

Eddington's (1915) paper that studies the ellipsoidal hypothesis is one of his great ones. We can do no better than use Eddington's own words:

"At any point of the system, the directions of the axes of the velocity ellipsoid determine three directions at right angles. The velocity ellipsoids thus define three orthogonal families of curves, each curve being traced by moving step by step always in the direction of an axis of the velocity ellipsoid at the point reached. These curves may be regarded as the intersections of a triply orthogonal family of surfaces, which we shall call the principal

velocity surfaces. The axes of the velocity ellipsoid at any point are normals to the three principal velocity surfaces through any point".

In modern language, the theory of collisionless systems such as galaxies begins with the Boltzmann equation:

$$
\frac{\partial F}{\partial t}+\mathbf{v} \cdot \frac{\partial F}{\partial \mathbf{x}}-\frac{\partial \Phi}{\partial \mathbf{x}} \cdot \frac{\partial F}{\partial \mathbf{v}}=0
$$

where $F$ is the phase space distribution function and $\Phi$ is the gravitational potential. At every point in the galaxy, we can define a velocity dispersion tensor

$$
\sigma_{i j}=\left\langle\left(v_{i}-\left\langle v_{i}\right\rangle\right)\left(v_{j}-\left\langle v_{j}\right\rangle\right)\right.
$$

where angled brackets denote averages over the distribution function. The velocity dispersion tensor $\sigma_{i j}$ is real and symmetric, and therefore by a well-known theorem in linear algebra has mutually orthogonal eigenvectors. Eddington is asserting that the integral curves of the eigenvectors provide a one-dimensional foliation into surfaces, which he calls the principal velocity surfaces. We shall return to the assumptions underlying this assertion shortly, as it is the precisely the point that troubled Chandrasekhar.

Eddington then shows via Lagrange's equations that a steady state distribution of stars moving in a gravitational potential $\Phi$ necessarily generates principal velocity surfaces that are confocal quadrics. Labelling the quadric surfaces by $(\lambda, \mu, \nu)$, these are recognised as ellipsoidal coordinates (e.g., Morse \& Feshbach 1953). Eddington now proves two further theorems. First, suppose that the distribution of velocities has exactly the Schwarzschild (1908) or triaxial Gaussian form

$$
F \propto \exp \left(-\frac{v_{\lambda}^{2}}{2 \sigma_{\lambda}^{2}}-\frac{v_{\mu}^{2}}{2 \sigma_{\mu}^{2}}-\frac{v_{\nu}^{2}}{2 \sigma_{\nu}^{2}}\right),
$$

where $\left(v_{\lambda}, v_{\mu}, v_{\nu}\right)$ are velocity components referred to the locally orthogonal axes and $\left(\sigma_{\lambda}, \sigma_{\mu}, \sigma_{\nu}\right)$ are the semiaxes of the velocity ellipsoid. This is the ellipsoidal hypothesis. Eddington showed that the only solutions for the principal velocity surfaces are spheres. However, the gravitational potential need not be spherical, but can take the general form

$$
\Phi(r, \theta, \phi)=f(r)+\frac{g(\theta)}{r^{2}}+\frac{h(\phi)}{r^{2} \sin ^{2} \theta},
$$


where $f, g$ and $h$ are arbitrary functions of the indicated arguments. These have sometimes been called Eddington potentials in the astronomical literature.

Secondly, Eddington considered the more general case of a stellar population with an arbitrary distribution of velocities. Under the assumption of the existence of principal velocity surfaces, he showed that the potential can take the general form in ellipsoidal coordinates

$$
\Phi(\lambda, \mu, \nu)=\frac{f(\lambda)}{(\lambda-\mu)(\lambda-\nu)}+\frac{g(\mu)}{(\mu-\lambda)(\mu-\nu)}+\frac{h(\nu)}{(\nu-\mu)(\nu-\lambda)} .
$$

Eddington does not consider the fully triaxial case in detail, but he does study the degenerations of the ellipsoidal coordinates into spheroidal coordinates. Here, the stars have oblate or prolate density distributions, the principal velocity surfaces are prolate or oblate spheroids and the velocity dispersion tensor is in general anisotropic. This was the first attempt to build galaxy models using the separable potentials. Except in the spherical limit, Eddington did not write down the form of the integrals of motion, leaving that task to his student, G.L. Clark (1937).

Although Eddington's paper is not without its flaws, it turned out to be remarkably prescient, anticipating developments over half a century later.

\section{Chandrasekhar's Criticism}

In retrospect, Chandrasekhar's venture into stellar dynamics seems both natural and brave. It is natural, as it is an obvious progression of his interests in stellar structure and evolution. It is brave, as it strays onto territory that Eddington had already made his own. The discipline had been founded by two people - Eddington in his book Stellar Movements and the Structure of the Universe published in 1914, and Jeans in his 1917 Adams Prize essay, published somewhat later in 1919 as Problems of Cosmogony and Stellar Dynamics. Eddington and Jeans had dominated the subject over the 1920s, with fundamental contributions, including Jeans' theorem, the equations of stellar hydrodynamics (sometimes called the Jeans' equations), and Eddington's inversion formula for the distribution function of a spherical galaxy. Given Chandrasekhar's worsening relationship with Eddington over these years, his incursions into this field were almost inevitably opening up a second front.

Chandrasekhar $(1939,1940)$ announced his entry into the field with two gigantic papers on the ellipsoidal hypothesis (summarised in Chapters 3 and 4 of Principles of Stellar Dynamics which themselves occupy over a hundred pages). Right away, he detected an error in Eddington's paper. Chandrasekhar's criticism is worth quoting in full:

"The fallacy in Eddington's argumentation is clear. It is true that we can regard the directions of the principal axes of the velocity ellipsoid at any given point as being tangential to the three curves which intersect orthogonally at the point considered. But it is 
not generally true that we can regard these curves as the intersections of a triply orthogonal system of surfaces. Consequently, the notion of principal velocity surfaces introduces severe restrictions on the problem, which are wholly irrelevant and certainly unnecessary."

Here, Chandrasekhar is completely correct. Eddington assumed that the eigenvectors of the velocity dispersion tensor are the tangent vectors of a triply orthogonal system of surfaces. This is a sufficient, but not a necessary, consequence of the orthogonality of the eigenvectors of the dispersion tensor. Eddington (1943) himself conceded as much in his review of Chandrasekhar's book. Writing in the journal Nature, he stated:

"Chandrasekhar rightly points out a fallacy in a theorem which I gave in 1915 and the correction makes the conclusion less general than has hitherto been assumed. But he does not take the opportunity of restating the position. Presumably it is still true that in a steady system with axial symmetry, the velocity surfaces are confocal quadrics and transverse star streaming is necessarily excluded, but there is no mention of this".

Where did Chandrasekhar's insight lead ? Chandrasekhar first somewhat generalised the problem by asking for stellar dynamical models with distribution functions $F$ of the form

$$
F=F(Q),
$$

where $Q$ is a quadratic function of the velocities. The coefficients are arbitrary functions of position. More formally,

$$
Q=\mathbf{v} \cdot \mathbf{M}(\mathbf{x}) \cdot \mathbf{v}+N(\mathbf{x}),
$$

where $\mathbf{M}$ and $N$ are matrix and scalar functions of position. This is a generalized ellipsoidal hypothesis, as $Q$ and hence the phase space density $F$ is constant on ellipsoids in velocity space.

Chandrasekhar proceeds by substituting his ansatz for the distribution function into the Boltzmann equation and separating term by term in the powers of velocity. He extracts a set of 20 partial differential equations, which he reduces to 6 integrability conditions. Note that Chandrasekhar does not impose the Poisson equation, as he is interested in finding the conditions that a stellar population has a distribution function of ellipsoidal form in an externally imposed potential. He reaches a very surprising conclusion that for stellar systems in a steady state, the potential $\Phi$ must necessarily be characterised by helical symmetry. The case of axial symmetry is included as a special case.

In other words, using cylindrical polar coordinates $(R, \phi, z)$, Chandrasekhar asserts that the only solutions for the gravitational potential compatible with the generalised ellipsoidal hypothesis are

$$
\Phi=f(R, z+\alpha \phi),
$$

where $f$ is an arbitrary function of the indicated arguments and $\alpha$ is a constant (the reciprocal of the pitch of the helix). The integrals of motion are the energy $E$ and the 
generalisation of the angular momentum component, namely

$$
I=p_{\phi}-\alpha p_{z}
$$

where $p_{\phi}$ and $p_{z}$ are the canonical momenta conjugate to $\phi$ and $z$. Chandrasekhar then notes that such a potential can have bound orbits only if it is axisymmetric $(\alpha=0)$ and so he reaches his final conclusion. For stellar systems with differential motions, which are in steady states and of finite spatial extent, the potential $\Phi$ must necessarily be characterized by axial symmetry.

This is a strong claim (we shall shortly see that, like Eddington's work, it is not entirely correct). A surprising aspect is that, having realised that Eddington had introduced unnecessarily restrictive assumptions into the problem, Chandrasekhar is not troubled by that fact that his more general approach finds fewer solutions than Eddington - and indeed doesn't find the solutions with quadric principal velocity surfaces at all! Even more curiously, Chandrasekhar recognises that the phase space distribution $F$ is an integral of motion, quoting Whittaker's (1936) book on Analytical Dynamics as a reference. He therefore knows that his problem is exactly equivalent to seeking all potentials that admit integrals of motion quadratic in the velocities. But, this problem is also (partly) solved in Whittaker's book, which provides a derivation of the separable potentials in spheroidal coordinates, though not ellipsoidal, from the assumption of quadratic integrals.

A new result of Chandrasekhar is that he provides an explicit counter-example to Eddington's assumption. The helically symmetric systems indeed remain the only ones known to us which do not possess mutually orthogonal principal velocity surfaces, but do satisfy the ellipsoidal hypothesis. They are not of much astrophysical interest as they do not resemble galaxies, but they remain of considerable intellectual interest.

Another insight of Chandrasekhar that has proved its worth is his repeated emphasis on the principal of equivalence. By this, he means that if several different models can be found sharing the same gravitational potential, then a more complex model that does not satisfy the ellipsoidal hypothesis can be built by weighted linear superposition. This idea has often been exploited in modern times to build realistic models by superpositions of analytic distribution functions (e.g., Fricke 1952, Dejonghe 1989, Emsellem et al. 1999).

\section{A Modern Approach}

Let us now state and give the solution to Chandrasekhar's problem anew from the point

of view of a modern dynamicist. Jeans' theorem tells us that the distribution function of a collisionless system depends only on the globally defined, isolating, integrals of motion. It therefore follows that $Q$ must be an integral of motion. Chandrasekhar's problem is exactly equivalent to identifying all those potentials that admit integrals of motion at most quadratic in the velocities. This is a problem of widespread interest in both mathematics and physics, with an enormous literature and history. 
Integrals of motion that are linear in the velocities always result from geometric symmetries of space. This is sometimes called Noether's theorem (see e.g., Landau \& Lifshitz 1976, Arnold 1978). It follows from the fact that the Lagrangian is invariant with respect to the corresponding transformations, which are linear in the generators of the Euclidean group of symmetries. Examples include the invariance of the angular momentum component $p_{\phi}$ in axisymmetric potentials $\Phi(R, z)$, and the invariance of the linear momentum component $p_{z}$ in translationally invariant potentials $\Phi(x, y)$. Chandrasekhar's helical solution is the most general possible, with rotationally and translationally invariant potentials given by the limits $\alpha \rightarrow 0$ and $\alpha \rightarrow \infty$ respectively.

Integrals of motion that are quadratic in the velocities always result from separability of the Hamilton-Jacobi equation in some coordinate system. Many authors discovered some or all of the potentials for which the Hamilton-Jacobi equation is separable in the confocal ellipsoidal coordinates or their degenerations (e.g., Eddington 1915, Weinacht 1924, Whittaker 1936, Clark 1937, Eisenhart 1948, Lynden-Bell 1962). These systems possess integrals of the motion quadratic in the velocities by construction, as the Hamilton-Jacobi equation only has such terms in it! The fact that separability of the Hamilton-Jacobi equation is both a necessary and sufficient condition is a much more difficult result to prove. It was done for the first time in Makarov et al. (1967).

Although written from the viewpoint of particle physicists, Makarov et al. (1967) follow essentially the same route as Chandrasekhar in Chapter 3 of Principles of Stellar Dynamics. That is, they ask for the Poisson bracket of the integral of motion $Q$ with the Hamiltonian $H$ to vanish. This is mathematically identical to requiring that $Q$ satisfy the collisionless Boltzmann equation, as Chandrasekhar did. The main difference is that Makarov et al. substantially simplify $Q$ by rotations and translations, before requiring that $Q$ commute with the Hamiltonian $H$. This considerably reduces the mathematical complexity of the problem, enabling them to find all possible solutions (including the separable ones that Chandrasekhar had missed).

Before passing to later developments, it is worth remembering that Chandrasekhar and Eddington had disagreed over the white dwarf stars and the endpoints of stellar evolution (see Vibert Douglas 1956, Wali 1996, Chandrasekhar 1988 for various perspectives on this affair). In retrospect, it is clear that Eddington behaved badly over the white dwarfs, not so much because he was wrong - that can (and should) happen to every scientist! - but because he used his seniority to stifle the work of a younger colleague.

Is it possible that Chandrasekhar, hurt by the reception of what would ultimately prove to be a Nobel Prize winning achievement, was unable to appreciate fully the advantages in Eddington's approach in stellar dynamics? True, he had detected an error in Eddington's (1915) paper, but Eddington in the end saw closer to the truth of the matter in stellar dynamics. Eddington introduced a hypothesis - the principal velocity surfaces - that was not strictly-speaking necessary and would ultimately be discarded by later scientists. But, it proved to be a physically fruitful hypothesis that led Eddington 
to an important class of models. Consequently, later developments were to build more on Eddington's work than Chandrasekhar's, as we will now see.

\section{Later Developments}

Further advances had to wait till the late fifties and early sixties, when the subject was revived by Lynden-Bell (1962) with a particularly original investigation. Rather than starting from an assumption that the integrals are quadratic in the velocities, LyndenBell permitted the integrals to have any form (polynomial or transcendent). Instead, he assumed that the steady-state is one of a set through which the system may secularly evolve whilst preserving the existence of the integrals of motion. This led to the enumeration of all potentials with such isolating integrals - prominent among them being the separable potentials in ellipsoidal coordinates and their degenerations. At the time, the flattening of elliptical galaxies was believed to be caused primarily by rotation rather than velocity anisotropy. Hence, the application of the potentials to galaxies remained unexplored in the West.

This was not true in the former Soviet Union, as a remarkable and sadly neglected paper by Kuzmin (1957) - citing the influences of Eddington (1915) and Clark (1937) had already used the separable potentials in spheroidal coordinates to build an oblate, axisymmetric model of the Galaxy. Kuzmin (1973) was also the first to write down the fully triaxial case, and study its orbital structure, identifying the 4 characteristic classes of orbits: box, inner and outer long axis tubes and short axis tubes (see e.g., Binney \& Tremaine 1987). These models became well-known in the West only after they had been re-discovered and extended by de Zeeuw (1985). Kuzmin (1973) and de Zeeuw (1985) showed that an ellipsoidally stratified model with density

$$
\rho=\frac{\rho_{0}}{\left(1+m^{2}\right)^{2}}, \quad m^{2}=\frac{x^{2}}{a^{2}}+\frac{y^{2}}{b^{2}}+\frac{z^{2}}{c^{2}}
$$

possesses an exactly separable gravitational potential in confocal ellipsoidal coordinates. The easiest way to demonstrate this is by making use of the methods and formulae in Chandrasekhar's (1968) finest and most beautiful book, Ellipsoidal Figures of Equilibrium. This is an important result as it showed that realistic and phyically motivated models of elliptical galaxies could be built from separable potentials. De Zeeuw also demonstrated a number of beautiful properties of these models, including the classification of their orbits in integral and action space ${ }^{1}$. This led to a flowering of interest

\footnotetext{
${ }^{1}$ By now, these potentials had come to be known as Stäckel potentials in the astronomical literature. This seems unwarranted. First, it is poor practice in physics to associate a name with an equation if a perfectly adequate descriptive term exists. On these grounds alone, the term 'separable potential' is preferable to 'Stäckel potential'. And, second, there is no reason to associate the name of Paul Stäckel with coordinate systems and potentials that he never wrote down! Stäckel was a prominent differential geometer, latterly Professor of Mathematics at Heidelberg. In his Habilitationschrift in 1891 at Halle,
} 
in the models, as evidenced by the papers in IAU Symposium 12\%: The Structure and Dynamics of Elliptical Galaxies (de Zeeuw 1987). This can be seen as the culmination of over seventy years of astronomical research on the subject, from Eddington, through Chandrasekhar, Lynden-Bell and Kuzmin to modern times.

Even though their mass density falls off faster than the luminosity density of giant ellipticals, and even though they are cored in the central parts rather than cusped, the separable models still occupy a special place in modern galactic dynamics. This is because the orbital structure of the models is generic for all flattened triaxial systems without figure rotation. Although the models do not contain any irregular or chaotic orbits, for many applications in galactic dynamics, this is unimportant, as the fraction of phase space occupied by truly irregular orbits is believed to be small (Goodman \& Schwarzschild 1981).

\section{The Alignment of the Velocity Dispersion Tensor}

Modern interest in the subject (e.g., Smith, Evans \& An 2009a,b, Binney \& McMillan 2011) has been given additional impetus by large-scale photometric and spectroscopic surveys of hundreds of thousands of stars in the Milky Way Galaxy itself. If proper motions are also available, then this raises the possibility that all the components of the velocity dispersion tensor can be computed directly from the data. There have been a number of interesting recent attempts to do this, both for halo and disk populations. Although sample sizes are presently still small, and distance errors a serious hazard, matters will substantially improve in the next few years.

For example, the Sloan Digital Sky Survey (SDSS, York et al. 2000) carried out repeated photometric measurements in an equatorial stripe, known as Stripe 82, primarily with the aim of supernova detection. Bramich et al. (2008) then provided a public archive of light-motion curves in Stripe 82 complete down to magnitude 21.5 in the $u, g, r$ and $i$ photometric bands, and to magnitude 20.5 in $z$. This reaches almost 2 magnitudes fainter than the SDSS/USNO-B catalogue (Munn et al. 2004), making it the deepest large-area photometric and astrometric catalogue available. Smith et al. (2009a,b) extracted a sample of $\sim 1,600$ halo subdwarf stars via a reduced proper motion diagram. Their radial velocities are calculated from the SDSS spectra and their distances are estimated from

Stäckel wrote down the condition for the Hamilton-Jacobi equation to separate in a given coordinate system on a general Riemannian manifold in the form of the vanishing of a determinant (which has reasonably enough come to be called the Stäckel determinant). Stäckel did not derive the coordinate systems in Euclidean 3-space for which his determinant vanishes, far less the form of the separable potentials in these coordinates. This work was left to Weinacht (1924) and Eisenhart (1948). In fact, Stäckel's result is limited, as it does not even provide a comprehensive test for separability. Stäckel's determinant for a separable system only vanishes if it is written down in the separable coordinate system itself. The finding of a general criterion for identifying whether a potential is separable in some coordinate system remains an outstanding research problem. 

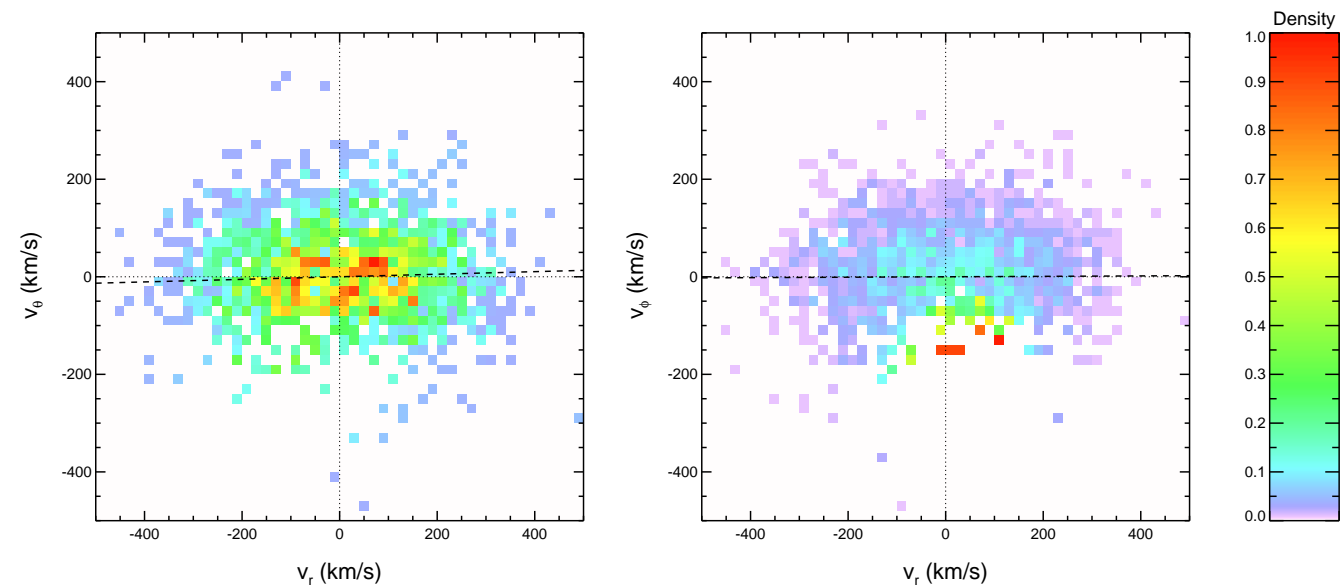

Figure 1. The efficiency corrected velocity distributions in the $\left(v_{r}, v_{\theta}\right)$ and $\left(v_{r}, v_{\phi}\right)$ planes for the sample of 1,600 subdwarfs with $1 \mathrm{kpc}<|z|<4 \mathrm{kpc}$. The dashed lines show the orientation of the tilts, which are very close to spherical alignment. The apparent non-Gaussianity in the $\left(v_{r}, v_{\phi}\right)$ distribution is due to the variation of the efficiency correction across this plane. [From Smith et al. (2009b)].

photometric parallaxes, thus giving the full phase space information. Although the sample is not kinematically unbiased, the detection efficiency can be calculated and corrections made for any biases.

Figure 1 shows the velocity distributions of the SDSS Stripe 82 subdwarfs. These halo stars lie at Galactocentric cylindrical polar radii between 7 and $10 \mathrm{kpc}$, and at depths of $4.5 \mathrm{kpc}$ or less below the Galactic plane. The good alignment of the velocity ellipsoid in spherical polars is already apparent from the velocity distributions in the $\left(v_{r}, v_{\theta}\right)$ and $\left(v_{r}, v_{\phi}\right)$ planes. Smith et al. find that the velocity dispersion tensor of the halo subdwarfs has semiaxes $\left(\sigma_{r}, \sigma_{\phi}, \sigma_{\theta}\right)=(143 \pm 2,82 \pm 2,77 \pm 2) \mathrm{kms}^{-1}$. The misalignment from the spherical polar coordinate surfaces can then be described by the correlation coefficients and the tilt angles using

$$
\operatorname{Corr}\left[v_{i}, v_{j}\right]=\frac{\sigma_{i j}^{2}}{\left(\sigma_{i i}^{2} \sigma_{j j}^{2}\right)^{1 / 2}},
$$

and

$$
\tan \left(2 \alpha_{i j}\right)=\frac{2 \sigma_{i j}^{2}}{\sigma_{i i}^{2}-\sigma_{j j}^{2}} .
$$

The tilt of the velocity ellipsoid with respect to the spherical polar coordinate system is found to be consistent with zero for two of the three tilt angles, and very small for the 
third. Specifically, Smith et al find:

$$
\begin{aligned}
& \operatorname{Corr}\left[v_{r}, v_{\theta}\right]=0.078 \pm 0.029, \quad \alpha_{r \theta}=3.4 \pm 1.3, \\
& \operatorname{Corr}\left[v_{r}, v_{\phi}\right]=-0.028 \pm 0.039, \quad \alpha_{r \phi}=-2.2 \pm 3.3, \\
& \operatorname{Corr}\left[v_{\phi}, v_{\theta}\right]=-0.087 \pm 0.047, \quad \alpha_{\phi \theta}=-37^{\circ} .4 \pm 20{ }^{\circ} .4 .
\end{aligned}
$$

In Eddington's language, these stars have spherical principal velocity surfaces to an excellent approximation. In a slight extension of the earlier results of Eddington (1915) and Chandrasekhar (1939), Smith et al. (2009b) prove that: If the potential is nonsingular, it is a sufficient condition for a spherical symmetric potential that one of the non-degenerate eigenvectors of the velocity dispersion tensor is aligned radially everywhere.

Of course, Smith et al. (2009b) did not demonstrate that the velocity dispersion tensor is aligned everywhere in spherical polar coordinates. They showed that the alignment is very close to spherical for halo subdwarfs at heliocentric distances of $<5 \mathrm{kpc}$ along the $\sim 250 \mathrm{deg}^{2}$ covered by SDSS Stripe 82. Nonetheless, they argued that this is still a striking and unexpected result over a range of Galactic locations that provides a new line of attack on the awkward question of the shape of the Milky Way's dark halo. Binney \& McMillan (2011) concur that local measurements are not enough to constrain the shape of the Galaxy's potential. Further work on the alignment of the velocity ellipsoid of halo populations is highly desirable.

By contrast, the behaviour of the velocity ellipsoid of disk populations has been more widely studied, not least because of its importance for calculations of the asymmetric drift and the Oort Limit. Based on evidence from orbit integrations, Binney \& Tremaine (1987) suggest that the tilt may lie midway between spherical and cylindrical polar alignment. This is also the expectation from models based on potentials separable in spheroidal coordinates (Statler 1989). There have been three recent determinations directly from data by Siebert et al. (2008), Fuchs et al. (2009) and Smith, Evans \& Whiteoak (2011).

Siebert et al. (2008) extracted 763 red clump stars from the Radial Velocity Experiment dataset (RAVE, Zwitter et al. 2008), spanning a distance interval from the Sun of 500 to 1500 pc. The tilt of the velocity ellipsoid of stars so close to the Galactic plane is affected both by the structure of the Galactic disk and and the flattening of the dark halo. Siebert et al. find that the velocity ellipsoid is tilted towards the Galactic plane with an inclination of $7^{\circ} .3 \pm 1.8$. This is entirely consistent with alignment in spherical polar coordinates. Siebert et al. compare this value to computed inclinations for two mass models of the Milky Way. The measurement is consistent with a short scalelength of the stellar disc $(\approx 2 \mathrm{kpc})$ if the dark halo is oblate or with a long scalelength $(\approx 3 \mathrm{kpc})$ if the dark halo is spherical or prolate.

Fuchs et al. (2009) used an enormous sample of $\sim 2$ million M dwarfs derived from the Sloan Digital Sky Survey Data Release 7 (Abazajian et al. 2008). Although the proper motions and photometric distances of these stars are available, unfortunately the radial velocities are not. Fuchs et al. estimated the radial velocities via the method of 

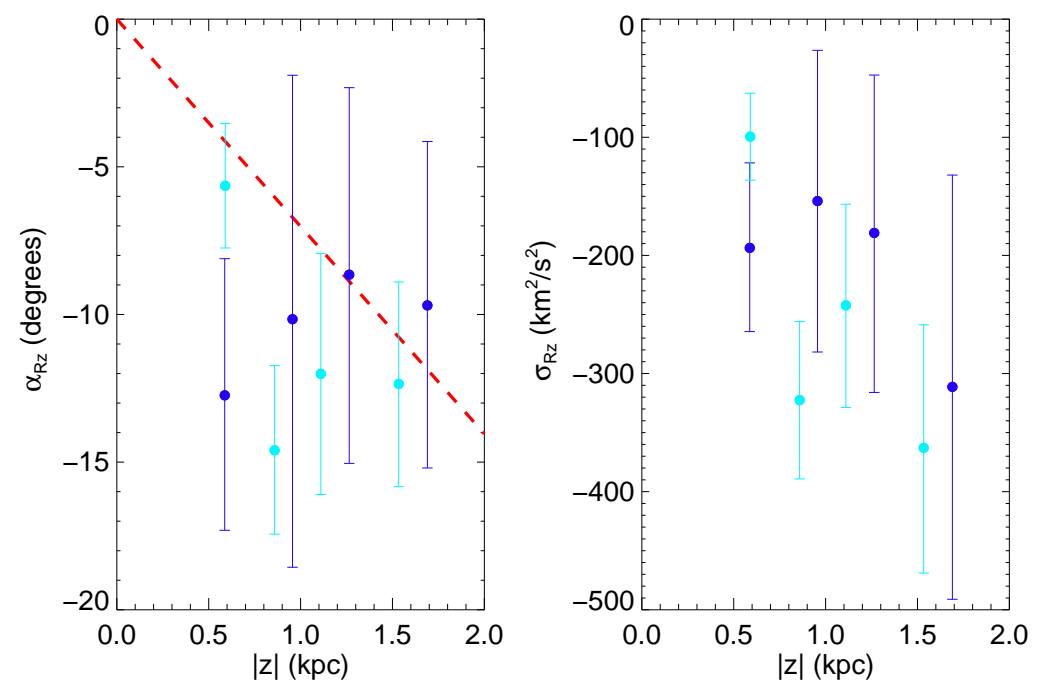

Figure 2. The variation of $\sigma_{R z}$ and the corresponding angle $\alpha_{R z}$ as a function of height from the plane. The dashed red line is the assumed halo tilt (i.e. aligned in spherical polars). The blue and cyan points correspond to disc stars with metallicities $-0.8 \leq[\mathrm{Fe} / \mathrm{H}] \leq-0.5$ and $[\mathrm{Fe} / \mathrm{H}] \geq-0.5$, respectively. [From Smith et al. (2011)].

deprojection of proper motions. They found an anomalously large tilt reaching an inclination of $20^{\circ}$ at heights above the Galactic plane of $800 \mathrm{pc}$, whereas spherical alignment would predict an inclination of $\approx 5^{\circ}$. McMillan \& Binney $(2009)$ have argued that this surprisingly large value may be spurious, a consequence of correlations between velocities and positions of stars, which renders the method of deprojection invalid.

Finally, Smith et al. (2011) again use the very deep light-motion catalogue for Stripe 82 (Bramich et al. 2008) to extract a sample of disk stars, complete with radial velocities from SDSS spectra and photometric metallicities. These stars are confined to a narrow range of cylindrical polar radius between $7 \leq R \leq 9 \mathrm{kpc}$. However, there are enough stars to split the data into three ranges in metallicity $(-1.5 \leq[\mathrm{Fe} / \mathrm{H}] \leq-0.8,-0.8 \leq$ $[\mathrm{Fe} / \mathrm{H}] \leq-0.5$ and $-0.5 \leq[\mathrm{Fe} / \mathrm{H}])$, and for each metallicity bin to divide the data into four ranges in $\mathrm{z}(0 \leq|\mathrm{z}| \leq 0.8,0.8 \leq|\mathrm{z}| \leq 1.1,1.1 \leq|\mathrm{z}| \leq 1.5$ and $1.5 \leq|\mathrm{z}| \leq 2.2 \mathrm{kpc})$. This gives around 500 to 800 stars per bin. The variation with height and metallicity is shown in Figure 2. The dotted line corresponds to what we would expect for a velocity ellipsoid aligned in spherical polar coordinates. The metal-rich and medium-metallicity stars are arguably consistent with the dotted line, and hence consistent with the result of 
Siebert (2008). In general, the stars in the lowest metallicity bins (not plotted in Figure 2 ) exhibit tilt angles which are larger than this, albeit with very noisy error bars.

Fortunately, the very-near future sees the dawning of the Age of Precision Astrometry. The GAIA satellite (e.g., Gilmore 2007) will provide tangential velocities for 44 million stars and distances for 21 million stars with an accuracy better than 1 per cent. There is therefore a realistic prospect that the behaviour of the velocity ellipsoid for both disk and halo populations over a swathe of locations in the Milky Way Galaxy will be known shortly.

\section{References}

Abazajian K., 2009 ApJS, 182, 543

Arnold V.I., 1978, Mathematical Methods of Classical Mechanics, Springer

Binney J., Tremaine S., 1987, Galactic Dynamics, Princeton

Binney J., McMillan P., 2011, MNRAS, submitted (arXiv:1101.0747)

Bramich D., et al., 2008, MNRAS, 386, 887

Chandraskehar S., 1939 ApJ, 90, 1

Chandraskehar S., 1940 ApJ, 92, 441

Chandrasekhar S., 1943 Principles of Stellar Dynamics, University of Chicago Press

Chandrasekhar S., 1968 Ellipsoidal Figures of Equilibrium, University of Yale Press

Chandrasekhar S., 1984 On Stars, Their Evolution and Their Stability, Nobel Foundation

Chandrasekhar S., 1988 Eddington: The Most Distinguished Astrophysicist of his Time, Cambridge University Press

Clark G.L., 1937 MNRAS, 97, 182

de Zeeuw P.T., 1985 MNRAS, 216, 273

de Zeeuw P.T., 1987 IAU Symposium 12\%: The Structure and Dynamics of Elliptical Galaxies, Reidel

Dejonghe H., 1989, ApJ, 343, 113

Eddington A.S., 1914 Stellar Movements and the Structure of the Universe, Macmillan.

Eddington A.S., 1915 MNRAS, 76, 37

Eddington A.S., 1943 Nature, 151, 91

Eisenhart L.P., 1948 Phys. Rev, 74, 87

Emsellem E., Dejonghe H., Bacon R,. 1999 MNRAS, 303, 495

Fricke W., 1952 Ast Nach., 280, 193

Fuchs B., 2009, AJ, 137, 4149

Gilmore G., 2007, In Exploring the Cosmic Frontier: Astrophysical Instruments for the 21st Century., p. 205

Goodman J., Schwarzschild M., 1981 Ap J, 245, 1087

Jeans J.H., 1919 Problems of Cosmogony and Stellar Dynamics, Cambridge University Press

Kuzmin G.G., 1956 Astr. Zh, 33, 27

Kuzmin G.G., 1973 In Dynamics of Galaxies and Clusters, Materials of the All Union Conference in Alma Ata, p. 71, English translation in de Zeeuw (1987)

Landau L.D., Lifshitz E.M., 1976, Mechanics, Pergamon

Lynden-Bell D., 1962 MNRAS, 124, 95

Makarov A.A., Smorodinsk J.A., Valiev Kh., Winternitz P., 1967 Nuovo Cimento, 52, 1061

McMillan P., Binney J., 2009 MNRAS, 400, L103 
Morse P., Feschbach H., 1953 Methods of Mathematical Physics, McGraw-Hill

Munn J.A., 2004, AJ, 127, 3034

Schwarzschild K., 1908 Göttingen Nachriten, p.191

Siebert A., 2009 MNRAS, 391, 793

Smith M.C., et al. 2009a, MNRAS, 399, 1223

Smith M.C., Evans N.W., An J.H. 2009b, Ap J, 698, 1110

Smith M.C., Evans N.W., Whiteoak H., 2011, MNRAS, submitted.

Stäckel P., 1891, Uber die Integration der Hamilton-Jacobischen Differential Gleichunger mittelst Separation der Variabeln, Habilitationschrift, Halle

Statler T.S, 1989, ApJ, 375, 544

Vibert Douglas A., 1956, The Life of Arthur Stanley Eddington, Nelson

Wali K., 1984, Chandra, University of Chicago Press

Weinacht J., 1924, Math Ann, 91, 279

Whittaker E.T., 1936 Analytical Dynamics, Cambridge University Press

York D., et al. 2000, AJ, 120, 1579

Zwitter T., et al. 2008, AJ, 136, 421

\section{Acknowledgements}

I am grateful to Donald Lynden-Bell and Tim de Zeeuw for first introducing me to this fascinating subject. I thank too my collaborators Jin An, Martin Smith and Hannah Whiteoak for their help and insight, as well as permission to report results in advance of publication. 